\title{
Scientific Viewpoints with Emphasis on Dermal Cellular Regeneration in Wound Sites
}

\author{
Denis E. Solomon \\ 9B Somerville Road, Wigan, England \\ Email: denissolomon@yahoo.com \\ Received 1 February 2014; revised 1 March 2014; accepted 8 March 2014 \\ Copyright (C) 2014 by author and Scientific Research Publishing Inc. \\ This work is licensed under the Creative Commons Attribution International License (CC BY). \\ http://creativecommons.org/licenses/by/4.0/

(c) $\underset{\mathrm{EY}}{\mathrm{B}}$ Open Access

\begin{abstract}
The human dermis presents an ongoing problem for regenerative medicine. Current medical management uses various acellular dermal matrices on wound sites. The challenge for scientists is to examine, then to question accepted conventional wisdom and to present new concepts. In this paper, Autologous Cell Therapy will be described by using cell culture of autologous dermal fibroblasts and their extracellular matrix as a foundation for rebuilding the dermis in conditioned wound beds*. This proposal seems to create a conflict with the medical approach to keeping a wound bed "moist".
\end{abstract}

\section{Keywords}

Autologous Cell Therapy; Dermal Regeneration; Dermal Fibroblast Cell Culture Techniques; Moist Wound Beds; Wound Healing

\section{Introduction}

While clinicians dictate a mode of wound care treatment, a scientist seeks an understanding of cellular processes occurring under the designated applied dressing. The viewpoints being described below have been developed by using past review papers [1]-[3] as a framework. Enormous strides have been made in wound bed preparation [4] [5], and the recognition of a biofilm has made the consideration of sluicing a wound bed a viable option [6]. There has been a medical debate about keeping a wound bed either "dry" or "moist". Currently, the consensus is to keep it “moist” based on Winter's 1962 [7] observations, using no human tissue but a young pig’s skin. Scientific findings will be presented below which dares to question this dictum.

\footnotetext{
*Also see: Yarwood-Ross L, Dignon AM. (2012) NPWT and moist wound dressings in the treatment of the diabetic foot. Br. J. Nurs. 21(15):S26, S28, S30-2.
} 
When this author published an initial, simple paper on the endothelial extracellular matrix (ECM) in 1992 [8] based on commonality of cell secretions in seemingly un-associated human tissues (the umbilical cord vein and the vascular aorta), there were no ECM-designated laboratories, and Biotechnology, e.g. tissue engineering and tissue regeneration, was still gearing up to become an established medical/scientific industry.

In spite of modern progress, the proper rebuilding of the human dermis has not been structurally derived on a purely cellular basis. Acellular dermal matrices of various origins have been described which are essentially "sheet" materials and are essentially employed as wound covering material (See Widgerow 2012, Part 1 under acellular matrices for named products, [9]) [10]. One of the major firms (making "Dermagraft") proved unprofitable and was recently sold to Organogenesis Inc., at a major loss. Skin grafts even if they "take", partly due to plasmatic imbibition, do not result in the regeneration of the dermis. Pressing a finger into a former wound site reveals a depression which can be not immediately apparent at first sight.

This author has recently described the signature side-on packing configuration at confluence of human dermal reticular fibroblasts at an angled vertical in vitro, obtained by non-enzymatic isolation, using $20 \%$ foetal bovine serum (FBS) [11]. These cell cultures can be established by using a patient's own plasma. It is doubtful whether 3D-bioprinting for the regeneration of the dermis can be utilised because to enable this process, inherent dermal fibroblast cell-cell intercellular junctions will have to be ripped apart to get a suitable yield of single fibroblasts from both the papillary and reticular layer.

Undoubtedly, ongoing research will describe unequivocally that these two types of fibroblasts differ in their cell secretions and provide results that they also differ with the subset of dermal fibroblast populations lying deeper within the reticular dermis. This author has already recorded a commonality in secretions of epidermal and dermal cells, Table I [12], which will definitely be expanded. The epidermis and the dermis should be viewed as a continuum and not as separate entities. Initially, this was not the research trend in wound care. Instead, expensive laboratory-created cultured epithelial autografts (only one major type of epidermal cell in these CEAs: the keratinocyte) were used a burns treatment without regard for the underlying dermis and its varied cellular content. This haste to medically cover up a wound or burn has been described by Ovington 2007 [13] as "cover and conceal".

Various dressings have been described, with biotechnology companies putting on the market newer ones, for use in chronic wound treatment. Medical dogma, it would seem, leans in the direction of the "proper" manufactured dressing. This approach might be suitably applicable if the dressing helps with the management of a wound bed, but what should come after?

\section{Moist V. Dry Wound Beds}

As stated above, clinicians now dictate that the wound bed should be kept moist [7] [14]. However, considerations of the production of the dermal fibroblast ECM seem to oppose this dictum. Figure I in Widgerow's 2012 (Part I) article [9] on bioengineered matrices [15] [16] suggest that in a stiff wound bed, there is more ECM production than in a soft (interpreted here, perhaps naively, as being similar to "moist") wound bed. Hence the scientific question, why keep the wound bed "moist"?

Hinz 2006 [17] reported that dermal fibroblast contractile actin stress fibres do not form on very soft culture substrate hydrogels or in compliant collagen gels, hence hindering the restoration of the ECM in damaged tissue. Writing a review, Eckes et al. 2010 [16] state that in the early and late phases of wound healing, ECM protein function is the vital director of tissue repair. The answer to the posed question may still lie with a clinical compromise, if this scientific viewpoint were seconded. An initial "moist" wound bed followed by an air-"dry" wound bed (once the granulation tissue has taken hold) to enable the resident dermal fibroblasts to fully go to work.

\section{Conditioning the Wound Bed}

Biotechnology companies have not made use of the natural cell secretions, which include growth factors and cytokines to be found in conditioned medium (CM) of dermal fibroblast cell cultures derived from healthy subjects. If the wound bed is going to be kept moist, why not utilize these natural secretions from primary reticular fibroblasts (RF) which can be admixed with the patient's own plasma and applied, even sprayed, onto the prepared wound bed, without patient discomfort?

This will be an attempt to "swamp" serum factors oozing from the exposed surface of the wound site which does not occur in intact, un-wounded tissue. 


\section{Scientific Proposal}

The above forms part of a radical proposal of "concreting over" the active wound site (especially chronic wound sites) with 1) autologous plasma and RF CM, 2) "live” reticular fibroblast ECM, 3) a mixture of "live” clusters of reticular fibroblasts and ECM to try to dampen and/or dowse the continuing inflammatory response in the wound bed.

The theoretical aspect of this proposal holds that seepage downwards from the newly applied autologous material may cause some unblocking of the existing status quo within the wound bed.

This innovative procedure has not been considered for a number of practical reasons. Firstly, pure reticular fibroblast cell isolation has been a scientific problem. Unless a segment of skin tissue (after stripping off the epidermis with a pair of forceps) was specifically cut with a dermatome, and followed by enzyme use or the setting up of a classic "explant” cell culture, scientists would obtain a mixture of papillary and reticular fibroblasts after enzyme use on minced dermis or the loose spread of fibroblasts from the explant which lacked the side-on packing configuration at an angled vertical earlier described for primary reticular fibroblasts at confluence [11]. For years, clinicians have treated the wound site with a variety of dressings which have become more sophisticated and expensive e.g. Promogran Prisma ${ }^{\mathrm{TM}}$, while scientists have steadily documented the in situ overproduction of inflammatory cytokines, metalloproteinases and the impedance of inherent growth factors et cetera (see reference lists in [1]-[3]). Solid advances in wound care have been few and far between. Also it has been reported that fibroblasts do not react to chronic wound fluid [18]; neonatal skin fibroblasts were used in this study which needs updating by repetition with adult reticular fibroblasts.

\section{Cellular Reconstruction}

Obviously, in wound beds and particularly those in chronic wounds, the papillary dermis has been denuded; using collected CM from autologous reticular fibroblasts would seem to present a natural first step in rebuilding the dermis from the reticular dermis upwards from a purely scientific viewpoint.

An aliquot of dermal fibroblast CM will be mixed with autologous plasma to "condition" the wound bed. Secondly, fragments of the ECM will be applied. Thirdly, the mixture of "live” ECM fragments with "live" cell clusters of primary autologous reticular fibroblasts in CM (after use of a cell scraper on a confluent monolayer) together with $4 \%(\mathrm{v} / \mathrm{v})$ glucose should also be considered for topical use.

Much later on, under clinical direction, carefully mix autologous primary papillary fibroblasts and fragments of the ECM in CM, with $4 \%$ (v/v) glucose and apply to the wound bed.

Taken together, the scientific viewpoint being put forward for consideration is twofold. Applying primary fibroblasts/ECM/CM/glucose onto a conditioned wound bed (after the topical application of CM/plasma) seems at first hand, to be a much more straightforward and direct means of trying to induce in situ senescent or immobile fibroblasts within the wound bed to phenotypically transition to potent, mobile fibroblasts instead of incorporating fibroblasts within a manufactured synthetic dermal matrix.

Secondly, the reticular fibroblasts on topical application would be expected to exert some beneficial synergistic effect; because they have been cultured with autologous plasma, they will now find themselves (to put it simply) in familiar surroundings within the "conditioned" wound bed. In other words, the fibroblasts have simply been "pre-conditioned" for active duty within the wound bed.

Follow up with topical application of papillary fibroblasts/ECM/CM/glucose. Consequently, contractile myofibroblasts should soon discover newly laid down fibroblast ECM and reinstated fibroblast-matrix interactions, which are fundamental for the formation of granulation tissue [16] [17]. Rebuilding the epidermal component has already been described in an earlier publication [19].

This method of treatment would also be cost effective in the current period of budgetary restraint and belt tightening. A fuller description of possible cell culture techniques is described below.

\section{Cell Culture of Dermal Fibroblasts with Autologous Plasma}

The procedure below can be used separately with a monolayer of papillary or reticular fibroblasts at confluence [11], but instead of 20\% FBS, 10\% autologous plasma will be used with (or without) the culture medium (IMDM, Iscove's modified Dulbecco's medium or Medium 199). A cell scraper or if sub-culturing, 5 mM EDTA/DPBS $\mathrm{pH} 7.4$ on confluent monolayers may be used. 
1) A confluent layer of reticular dermal fibroblasts in a T-25 flask is approximately $6 \times 10^{6}$ cells. If $5 \mathrm{mM}$ EDTA/DPBS (Dulbecco's phosphate-buffered saline) pH 7.4 is used for subculture, cells clusters will be retracted, leaving an intact ECM in the flask. If transferred to a T-75 flask with culture medium and $10 \%$ plasma, the cells will attach at their own pace. DPBS will be used to wash the cell layer and the culture medium changed every 2 - 3 days until confluence. Collect conditioned medium (CM) in a $25 \mathrm{ml}$. centrifuge tube. Swirl manually, leave to stand for 10 minutes, and pipette off most of the volume of CM, leaving behind the last $1-2 \mathrm{ml}$. (with cell debris) at the bottom of the centrifuge tube.

A T-75 flask, at confluence contains approximately $10 \times 10^{6}$ cells. At confluence, $3 \mathrm{ml}$. of the CM is removed, leaving $4 \mathrm{ml}$. behind in the flask. For clinical use, a cell scraper is used immediately. To the fragments of the ECM and cell clusters in $4 \mathrm{ml}$. CM, 4\% (v/v) of glucose is added. This sticky mass of liquid and cells makes application to a prepared wound bed, a practical clinical option, because it will adhere. It could be simply pipetted slowly onto the wound bed, using a pipette with a large enough bore at its bottom tip to facilitate the unhindered efflux of intact cell clusters. I would use a $25 \mathrm{ml}$. plastic pipette. A syringe would cause some shredding of cell clusters and ECM fragments which would be totally counter-productive.

2) Returning to the ECM left behind in the flask, an alternative procedure may be adopted. Half of the volume containing the cell clusters obtained by retraction with $5 \mathrm{mM}$ EDTA/DPBS, can be re-seeded onto the ECM. They will attach rapidly, and time to confluence will be at least halved. The same procedure, used to seed human umbilical vein endothelial cells onto their ECM, is illustrated in Figure 2, [8]. If the ECM substrate is "wounded" by a cell scraper to create a small hole, it will be of interest to observe the morphology of the dermal fibroblasts migrating to fill that hole, because fibroblasts can change their focal adhesion points and display a phenotypic transition with a change of mechanical microenvironment [3]. Alternatively, do not "wound" the cell layer. The ECM left behind after the use of $5 \mathrm{mM}$ EDTA/DPBS will consist of two superimposed ECMs. Use a cell scraper to get "live" fragments of ECM for application to the wound bed.

3) Alternatively, a cell scraper could be used on the confluent monolayer in the original T-25 flask in $2 \mathrm{ml}$. of $\mathrm{CM}$. The yield will be fragments of the ECM plus cell clusters of primary dermal fibroblasts. Add $2 \mathrm{ml}$. of autologous platelet-rich plasma [20]. Add the required aliquot of $4 \%$ (v/v) glucose and apply to the wound site.

4) The steps 1 - 3 described above provide practical illustrations, that autologous cell therapy using primary un-manipulated cells is a viable option in wound care. Platelet-rich or -poor plasma may be used, dependent on clinical opinion. If more than one segment of autologous skin tissue is at hand, a T-75 flask could be used for the initial seeding of the dermal fibroblasts which would of course, vastly expand the cell yield even after a single sub-culture. It should be noted that fragments of ECM do not attach to a plastic substrate [8]. If a cell scraper with a broad base were used, larger cell clusters with a larger percentage of intact cell-cell intercellular junctions will be retained which would be distinctly advantageous for intended use. If after excavating deeper within the reticular dermal tissue with the scalpel blade, and microvascular endothelial cells, maybe $1 \%$ - $2 \%$, were morphologically recognised within the fibroblast cell culture, I would continue on to confluence and use the natural co-culture for application to the "conditioned" wound bed.

\section{Precursor Cell Culture Experiments}

Plasma, like serum when employed in cell culture, is usually heat-inactivated, but when the concomitant use of autologous plasma with autologous cells is contemplated, there really is no need to heat-inactivate. Informed opinion might disagree.

As a precursor, simple cell culture experiments could be performed using senescent fibroblasts separately and together from both the papillary and reticular dermal layers and exposing them to various quantities (e.g. 1 - 5 $\mathrm{ml}$.) of CM, 5\% - 20\% autologous plasma, and $4 \%(\mathrm{v} / \mathrm{v})$ glucose. These pilot experiments will provide a firm indication of the proper percentage of plasma to employ with primary and senescent fibroblasts in cell culture. Time to confluence will be monitored. However, for the time being, a straight forward swop of $10 \%$ plasma for the normal $20 \%$ foetal bovine serum (FBS) [11] is being put forward for use. The percentage of plasma will be used with or without the culture medium to determine the optimal criteria.

$4 \%(\mathrm{v} / \mathrm{v})$ glucose is chosen as a nutritional source for the cells based on its common medical use with saline as a hypotonic solution. It is being assumed that in a wound bed, normal nutritional channels may be clogged or even temporarily blocked, hence application of cells without a source of nutrition seems inadvisable. 


\section{Acquiring Autologous Skin Tissue}

With wounds in non-diabetic patients, would it be ethically acceptable to expand the wound site ever so slightly to allow previously undamaged tissue to supposedly take the lead in the healing process and possibly overlap into the wound site causing clinical difficulty? This would preserve the specific fibroblast gene pattern expression, referred to as an "anatomic site identity code" in a previous publication [11]. A postage stamp-sized segment of site-specific autologous skin tissue would be quite sufficient to cell culture both the epidermal cells and various dermal fibroblasts; additionally, acquiring both the fragments of ECM and the CM being proposed for use.

Harding et al. 2007 [2] have described in full detail, the benefit of whole body care before attending the presenting wound site and described the misdiagnosis of certain conditions. In the digital computer world we live in, would a (PDR) Physician Desk Reference Supplement containing photographs of wound sites with legends of: 1) Diagnosis 2) Treatment regime 3) Outcome be useful to attending Wound Care physicians? This proposed supplement could take the form of an encrypted app.

It should be noted that there has been no published reports of basic cell science experiments using mesenchymal stem cells and primary human dermal fibroblasts, isolated by a non-enzymatic cell culture technique [11].

\section{Conclusion}

In conclusion, the author is under no illusion that the above concept only presents a simplistic approach to complex clinical problems. Secreted cell-based materials and the cells themselves (Autologous Cell Therapy) may not yet provide some answers at no major expense and with simple repetition of dermal fibroblast cell cultures and clinical application. The use of autologous cells to meet patients' needs does and will remain a thoughtful, timeless proposition.

\section{Conflict of Interest}

Since this is a proposal for medical treatment, there is none.

\section{References}

[1] Sarabahi, S. (2012) Recent Advances in Topical Wound Care. Indian Journal of Plastic Surgery, 45, 379-387. http://dx.doi.org/10.4103/0970-0358.101321

[2] Harding, K., Gray, D., Timmons, J. and Hurd, T. (2007) Evolution or Revolution? Adapting to Complexity in Wound Management. International Wound Journal, 4, 1-12. http://dx.doi.org/10.1111/j.1742-481X.2007.00329.x

[3] Hinz, B., Phan, S.H., Thannickal, V.J., Galli, A., Bochaton-Piallat, M.-L. and Gabbiani, G. (2007) The MyofibroblastOne Function, Multiple Origins. American Journal of Pathology, 170, 1807-1816. http://dx.doi.org/10.2353/ajpath.2007.070112

[4] Falanga, V. (2000) Classifications for Wound Bed Preparation and Stimulation of Chronic Wounds. Wound Repair and Regeneration, 8, 347-352. http://dx.doi.org/10.1111/j.1524-475X.2000.00347.X

[5] Schultz, G.S., Sibbald, G.R., Falangna, V., Ayello, E., Dowsett, C., Harding, K., Romanelli, M., Stacey, C., Teot, L. and Vanscheidt, W. (2003) Wound Bed Preparation: A Systematic Approach to Wound Management. Wound Repair and Regeneration, 11, S1-S28. http://dx.doi.org/10.1046/j.1524-475X.11.s2.1.X

[6] Klein, M.B., Hunter, S., Heimbach, D.M., Engrav, L.H., Honari, S., Gallery, E., Kiriluk, D.M. and Gibran, N.S. (2007) The Versajet Water Dissection: A New Tool for Tangential Excision. Journal of Burn Care \& Research, 26, 483-487. http://dx.doi.org/10.1097/01.bcr.0000185398.13095.c5

[7] Winter, G.D. (1962) Formation of Scab and the Rate of Epithelialisation of Superficial Wounds in the Skin of the Young Domestic Pig. Nature, 193, 293-294. http://dx.doi.org/10.1038/193293a0

[8] Solomon, D.E. (1992) The Seeding of Human Aortic Endothelial Cells on the Extra-Cellular Matrix of Human Umbilical Vein Endothelial Cells. International Journal of Experimental Pathology, 73, 491-501.

[9] Widgerow, A.D. (2012) Bioengineered Matrices Part 1. Attaining Structural Success in Biologic Skin Substitutes. Annals of Plastic Surgery, 68, 568-573. http://dx.doi.org/10.1097/SAP.0b013e31824b3d04

[10] Chen, S.-G., Tzeng, Y.-S. and Wang, C.-H. (2012) Treatment of Severe Burn with DermACELL®, an Acellular Dermal Matrix. International Journal of Burns and Trauma, 2, 105-109. 
[11] Solomon, D.E. (2013) Human Dermal Reticular Fibroblasts at Confluence Display a Signature Micro Pattern in Vitro. Open Journal of Regenerative Medicine, 2, 99-105. http://dx.doi.org/10.4236/ojrm.2013.24014

[12] Solomon, D.E. (2002) An in Vitro Examination of an Extracellular Matrix Scaffold for Use in Wound Healing. International Journal of Experimental Pathology, 83, 209-216. http://dx.doi.org/10.4236/ojrm.2013.24014

[13] Ovington, L. (2007) Advances in Wound Dressings. Clinics in Dermatology, 25, 33-38. http://dx.doi.org/10.1016/j.clindermatol.2006.09.003

[14] Cho, C.Y. and Lo, J.S. (1998) Excision and Repair: Dressing the Part. Clinics in Dermatology, 16, 25-47. http://dx.doi.org/10.1016/S0733-8635(05)70485-X

[15] Grinnell, F. (2003) Fibroblast Biology in Three-Dimensional Collagen Matrices. Trends in Cell Biology, 13, $264-269$. http://dx.doi.org/10.1016/S0962-8924(03)00057-6

[16] Eckes, B., Nischt, R. and Krieg, T. (2010) Cell-Matrix Interactions in Dermal Repair and Scarring. Fibrogenesis \& Tissue Repair, 3, 4. http://dx.doi.org/10.1186/1755-1536-3-4

[17] Hinz, B. (2006) Masters and Servants of the Force: The Role of Matrix Adhesions in Myofibroblast Force Perception and Transmission. European Journal of Cell Biology, 85, 175-181. http://dx.doi.org/10.1016/j.ejcb.2005.09.004

[18] Mendez, M.V., Raffetto, J.D., Phillips, T., Menzoian, J.O. and Park, H.Y. (1999) The Proliferative Capacity of Neonatal Skin Fibroblasts Is Reduced after Exposure to Venous Ulcer Wound Fluid: A Potential Mechanism for Senescence in Venous Ulcers. Journal of Vascular Surgery, 30, 734-743. http://dx.doi.org/10.1016/S0741-5214(99)70113-8

[19] Solomon, D.E. (2012) Mimicry of a Natural, Living Intra-Epidermal Micro Pattern Used in Guided Tissue Regeneration of the Human Epidermis. Journal of Developmental Biology and Tissue Engineering, 4, 1-7.

[20] Kushida, S., Kakudo, N., Suzuki, K. and Kusumoto, K. (2013) Effects of Platelet-Rich Plasma on Proliferation and Myofibroblastic Differentiation in Human Dermal Fibroblasts. Annals of Plastic Surgery, 71, 219-224. http://dx.doi.org/10.1097/SAP.0b013e31823cd7a4 